\title{
NONPERFECT SPACES WITH POINT-COUNTABLE BASES
}

\author{
PETER DAVIES ${ }^{1}$
}

\begin{abstract}
We construct a completely regular space of cardinality $\aleph_{1}$ with a point-countable base, which is not perfect. This answers a question of Fleissner and Reed. We also construct, under the hypothesis $2^{k_{0}}<2^{k_{1}}$, a hereditarily normal space of cardinality $\aleph_{1}$ with a $\sigma$-disjoint base, which is not perfect.
\end{abstract}

0. Introduction. In a preliminary version of their paper [FR] Fleissner and Reed ask the question: Is every regular space of cardinality $\aleph_{1}$ with a point-countable base perfect (every closed set is a $G_{\delta}$ )? We answer their question, negatively, with a completely regular counterexample. The example is constructed in two stages. First we construct a completely regular, first countable space containing a closed discrete subspace which is not a $G_{\delta}$. Then using the technique of "splitting points" employed by Bing [B], and made explicit by Tall [ $\left.\mathbf{T}_{1}\right]$, and Chaber [C], we construct the required space.

Fleissner and Reed show that if the hypothesis is strengthened to " $\sigma$-pointfinite base", then there is no absolute counterexample. They prove, under Martin's Axiom plus the negation of the continuum hypothesis, that all such spaces are perfect. We show that a counterexample with a $\sigma$-disjoint base exists, under the hypothesis that $2^{k_{0}}<2^{k_{1}}$. Thus, the statement that regular spaces of cardinality $\aleph_{1}$ with $\sigma$-point-finite bases are perfect is independent of and consistent with the usual axioms of set theory.

1. The examples. Space $X$. A completely regular, first countable space of cardinality $\aleph_{1}$ containing a closed discrete subspace which is not a $G_{\delta}$.

Let $X=\omega_{1} \times \omega_{1}$. Points off the diagonal or of the form $\langle\alpha, \alpha\rangle$, where $\alpha$ is a successor ordinal, are taken to be isolated. We construct a countable neighbourhood base for each of the remaining points as follows:

Let $\alpha<\omega_{1}$ be a limit ordinal, and let $\left\{\alpha_{n}\right\}_{n<\omega}$ be some increasing sequence, cofinal in $\alpha$. For every $\beta<\alpha$ let $U(\alpha, \beta)=\left(\alpha_{m}, \alpha\right) \times\{\beta\}$ where $m=\inf \left\{n: \beta<\alpha_{n}\right\} \quad\left(\left(\alpha_{n}, \alpha\right)\right.$ denotes the open interval in the usual order topology on $\left.\omega_{1}\right)$. For every $n<\omega$, let $V(\alpha, n)=\{\langle\alpha, \alpha\rangle\} \cup \cup_{\beta>\alpha_{n}} U(\alpha, \beta)$. $\{V(\alpha, n)\}_{n<\omega}$ is the countable neighbourhood base for $\langle\alpha, \alpha\rangle$.

It is not hard to see that $X$ is first countable, $T_{1}$ and zero-dimensional, and

Received by the editors December 19, 1979 and, in revised form, February 19, 1979.

AMS (MOS) subject classifications (1970). Primary 54D99, 54G20; Secondary 02K05.

Key words and phrases. Point-countable base, perfect.

${ }^{1}$ This research was supported by a National Research Council of Canada Postgraduate Scholarship. 
hence completely regular. The subset $\Delta=\left\{\langle\alpha, \alpha\rangle: \alpha<\omega_{1}, \lim (\alpha)\right\}$ is closed discrete. Suppose it were a $G_{\delta}$. Then $X \backslash \Delta=\cup_{n<\omega} C_{n}$ where for each $n<\omega$, $C_{n}$ is closed in $X$. For each $n<\omega$, let $A_{\beta}^{n}=\left\{\alpha:\langle\alpha, \beta\rangle \in C_{n}\right\}$ and $S_{n}=\{\beta$ : $A_{\beta}^{n}$ is unbounded in $\left.\omega_{1}\right\}$. Then for some $n<\omega, S_{n}$ is stationary in $\omega_{1}$. Let $\gamma_{0} \in S_{n}$ be arbitrary and, for $1<m<\omega$, inductively pick $\gamma_{m} \in S_{n} \cap$ $\bigcap_{i<m} \mathrm{cl}_{\omega_{1}} A_{\gamma_{i}}^{n}$ (closure in $\omega_{1}$ with the usual topology). Let $\gamma=\sup \left\{\gamma_{m}\right.$ : $m\langle\omega\}$. Then $\langle\gamma, \gamma\rangle \in \mathrm{cl}_{X} C_{n}=C_{n}$. Hence $C_{n} \cap \Delta \neq \varnothing$ a contradiction. Therefore $\Delta$ is not a $G_{\delta}$ in $X$.

Space $Y$. A completely regular space of cardinality $\aleph_{1}$ with a point-countable base, which is not perfect.

We prove a general lemma and then note that our space $X$ satisfies its hypothesis and thus generates our required space $Y$.

LEMMA. For every space $X$ which is

(a) of cardinality $\boldsymbol{}_{1}$,

(b) first countable, and,

(c) has a closed discrete subspace which is not a $G_{\delta}$,

there is a space $Y$ which is (a), (b) and (c), has a point-countable base, and has the same separation properties as $X$.

Proof. Let $X$ be as in the hypothesis, and $C \subset X$ a closed discrete subspace which is not a $G_{\delta}$. We can enumerate $C$ as $\left\{c_{\alpha}\right\}_{\alpha<\kappa}$ for some $\kappa<\omega_{1}$. Let $D=X \backslash C$. Let $D^{\#}=\left\{\langle d, \alpha\rangle: d \in D, \alpha<\omega_{1}\right\}$. Let $Y=C \cup D^{\#}$ with the following topology:

(i) Points of $D^{\#}$ are isolated.

(ii) For each $\alpha<\kappa$, pick a countable neighbourhood base (in $X$ ) for $c_{\alpha}$, say $\{M(\alpha, n)\}_{n<\omega}$, such that for each $n<\omega, M(\alpha, n) \cap(C \backslash\{\alpha\})=\varnothing$. Our neighbourhood base for $c_{\alpha}$ in $Y,\{N(\alpha, n)\}_{n<\omega}$, is defined by: for each $n<\omega$, $\left.N(\alpha, n)=\left\{c_{\alpha}\right\} \cup\{\langle d, \beta\rangle: d \in M(\alpha, n), \beta\rangle \alpha\right\}$. Clearly $|Y|=\aleph_{1}$. By the same arguments as in $\left[\mathrm{T}_{1}\right] Y$ has the same separation properties as $X$. A particular $\langle d, \beta\rangle$ can only be in some $N(\alpha, \beta)$ if $\alpha<\beta$. Therefore $Y$ has a point-countable base. Clearly $C$ is closed discrete in $Y$. Suppose $C$ is a $G_{\delta}$ in $Y$. Then $C=\bigcap_{n<\omega} O_{n}$ where each $O_{n}$ is open in $Y$. We can assume for each $n<\omega$ that $O_{n}=\cup_{\alpha<\kappa} N\left(\alpha, m_{\alpha}\right)$, where for all $\alpha<\kappa, m_{\alpha} \in \omega$. For each $n<\omega$ let $O_{n}^{\prime}=\cup_{\alpha<\kappa} M\left(\alpha, m_{\alpha}\right)$. Since $C$ is not a $G_{\delta}$ in $X$, there is some $d \in D$ such that $d \in O_{n}^{\prime}$ for every $n<\omega$. Hence for each $n<\omega$, $d \in$ $M\left(\alpha_{n}, m_{\alpha_{n}}\right)$ for some $\alpha_{n}<\kappa$ and $m_{\alpha_{n}}<\omega$. Let $\alpha=\sup \left\{\alpha_{n}: n<\omega\right\}$. Then $\langle d, \alpha\rangle \in N\left(\alpha_{n}, m_{\alpha_{n}}\right)$ for each $n<\omega$. Hence $\langle d, \alpha\rangle \in \bigcap_{n<\omega} O_{n}$, a contradiction. Therefore $C$ is not a $G_{\delta}$ in $Y$.

REMARKs. (1) $X$ is not normal (consider disjoint subsets of $\Delta$ corresponding to disjoint stationary subsets of $\omega_{1}$ ).

(2) Any space with the properties of the lemma (where the discrete subset is uncountable) cannot be collectionwise Hausdorff.

Therefore it is consistent with the axioms of set theory that no such space is 
normal (see $\left[\mathbf{T}_{2}\right]$ or $[\mathbf{F}]$ ), and so our construction cannot yield a normal (absolute) counterexample.

QUESTION. Does there exist a (real) normal space of cardinality $\aleph_{1}$ with a point-countable base which is not perfect? By the remarks above, if there is such a space, the closed set which is not a $G_{\delta}$ cannot be discrete.

Space $Z$. $\left(2^{\kappa_{0}}<2^{\kappa_{1}}\right)$. A space of cardinality $\aleph_{1}$ which is hereditarily normal, has a $\sigma$-disjoint base and is not perfect.

Let $Z$ be any subset of the reals of cardinality $\aleph_{1}$. Because $2^{\boldsymbol{k}_{0}}<2^{\boldsymbol{k}_{1}}$ implies there are no $Q$-sets, there exists a subset $W$ of $Z$ which is not an $F_{\sigma}$ in $Z$ (with the subspace topology). Extend the subspace topology on $Z$ by making points of $W$ isolated. This new topology is hereditarily normal and has a $\sigma$-disjoint base (see for example [E, p. 380]). Also $W$ is still not an $F_{\sigma}$ and, since it is open, witnesses that our new topology is not perfect.

\section{BIBLIOGRAPHY}

[B] R. H. Bing, A translation of the normal Moore space conjecture, Proc. Amer. Math. Soc. 16 (1965), 612-619.

[C] J. Chaber, Metacompactness and the class MOBI, Fund. Math. 91 (1976).

[E] R. Engelking, General topology, Polish Scientific Publishers, Warsaw, 1977.

[F] W. G. Fleissner, Normal Moore spaces in the constructible universe, Proc. Amer. Math. Soc. 46 (1974), 224-298.

[FR] W. G. Fleissner and G. M. Reed, Paralindelof spaces and spaces with a $\sigma$-locally countable base, Topology Proc. 2 (1977), 89-110.

[T, F. D. Tall, On the existence of normal metacompact Moore spaces which are not metrizable, Canad. J. Math. 26 (1974), 1-6.

$\left[\mathrm{T}_{2}\right]$, Set-theoretic consistency results and topological theorems concerning the normal Moore space conjecture and related problems, Thesis, University of Wisconsin, Madison, 1969; Dissertationes Math. 148 (1977), 1-53.

Department of Mathematics, University of Toronto, Toronto, Canada M5S-1A1 